\title{
The Effectiveness of Personal Sound Amplification Products in Adults With Mild to Moderate Hearing Loss: Is Their Use Inevitable?
}

\author{
Goun Choe (iD · Moo Kyun Park (i) \\ Department of Otorhinolaryngology-Head and Neck Surgery, Seoul National University College of Medicine, Seoul, Korea
}

The size of the global assistive device market is steadily increasing due to population aging and an increase in the elderly population with disabilities. The incidence of age-related hearing loss is also increasing, and accordingly, the global hearing aid market is also rapidly growing. However, fewer than $20 \%$ of adults with age-related hearing loss use a hearing aid, reflecting a considerable gap compared to the actual need. According to the Korean National Health and Nutrition Examination Survey, 30\% of the elderly have hearing loss, and $60 \%$ of them have mild to moderate hearing loss. If proper rehabilitation for hearing loss is not performed, it can lead to various real-world problems. Hearing loss causes difficulties in communicating with others, thereby contributing to social and psychological isolation among the elderly. In addition, the recent use of masks and social distancing due to coronavirus disease 2019 (COVID-19) have made communication increasingly difficult.

Personal sound amplification products (PSAPs) were originally designed to amplify sounds for people with normal hearing, and they are not medical devices. As such, PSAPs are not designed for hearing loss, but a growing number of people are using them for the purpose of solving hearing loss because of their easy access and low price. Mamo et al. [1] commented that the directto-consumer hearing device market serves as a stepping stone to hearing aid use. However, since PSAPs are devices that consumers directly control and use without an expert opinion, there is also concern about hearing damage due to unnecessary sound

- Received June 8, 2021

Accepted June 10, 2021

- Corresponding author: Moo Kyun Park

Department of Otorhinolaryngology-Head and Neck Surgery, Seoul

National University College of Medicine, 103 Daehak-ro, Jongno-gu,

Seoul 03080, Korea

Tel: +82-2-2072-2447, Fax: +82-2-745-2387

E-mail: aseptic@snu.ac.kr amplification.

As interest in PSAPs has increased, many studies have investigated their use. Reed et al. [2] compared hearing aids and five PSAPs in 42 adults with mild to moderate hearing loss, and four out of the five devices enabled improved speech understanding compared to the unaided condition, showing similar effects to hearing aids. Brody et al. [3] conducted a single-blinded, crossover study using three PSAPs and a traditional hearing aid in 25 elderly patients with mild to moderate hearing loss. All PSAPs showed improvement in speech recognition and listening effort compared to the unaided state, and the sound quality was similar to that of hearing aids. In a prospective cohort study comparing a PSAP, a basic hearing aid, and a premium hearing aid in 56 participants (19 with mild hearing loss, 20 with moderate hearing loss, and 17 with moderately severe hearing loss), the PSAP showed the same effects as the basic and premium hearing aids in the mild and moderate hearing loss groups [4]. Choi et al. [5] compared a PSAP and a basic hearing aid in 19 participants with mild to moderate hearing loss and stated that both devices provided considerable benefits for speech intelligibility in quiet situations. Kim et al. [6] conducted a pilot study comparing three basic PSAPs, three high-end PSAPs, and hearing aids in patients with moderate hearing loss. In that study, electroacoustic analysis and simulated real-ear measurements (REMs) were performed, and some PSAPs showed an adequate level of performance in these tests. In addition, in clinical experiments conducted later, PSAPs showed better results than the unaided conditions and similar results to hearing aids in the word recognition test and Korean version of the Hearing in Noise Test [6].

However, the above papers also presented limitations of PSAPs. Reed et al. [2] showed that one of the five PSAPs, which was very cheap, did not improve hearing. In 2019, the same research

Copyright $@ 2022$ by Korean Society of Otorhinolaryngology-Head and Neck Surgery

This is an open-access article distributed under the terms of the Creative Commons Attribution Non-Commercial License (https://creativecommons.org/licenses/by-nc/4.0)

which permits unrestricted non-commercial use, distribution, and reproduction in any medium, provided the original work is properly cited. 
group conducted a pilot study on a PSAP control protocol for nine patients with mild to moderate hearing loss. Although it was not clinically meaningful, when the audiologist adjusted the PSAP, better hearing results were found than as a result of the self-adjustment [7]. Brody et al. [3] also concluded that, in general, the hearing aid improved speech recognition performance and reduced listening effort significantly more than all PSAPs. Cho et al. [4] found that, in the moderately severe hearing loss group, the premium hearing aid showed good performance in most tests. Choi et al. [5] did not show improvement in speech intelligibility when a PSAP was used in a noisy environment, but wearing a hearing aid improved speech intelligibility with a noisy background. Kim et al. [6] showed that only two high-end PSAPs met all tolerances in terms of electroacoustic analysis. In the simulated REMs, one basic and two high-end PSAPs showed appropriate levels for three common hearing loss configurations. Additionally, a study explored Amazon customers' opinions of PSAPs, and the positive reviews showed that there was a strong correlation with the price of the product, demonstrating that PSAPs are also subject to financial influence [8].

In a 2015 report by President Obama's Council of Advisors on Science and Technology, untreated hearing loss was viewed as a major health and social problem, and the report focused on patients with mild to moderate age-related hearing loss and PSAPs. Accordingly, the US Congress passed the Over-the-Counter Hearing Aid Act of 2017 for people with mild to moderate hearing loss, but the FDA missed the August 18, 2020, statutory deadline during the COVID-19 pandemic. The need for nonmedical hearing amplification devices is emerging worldwide, but at the same time, there is no definite regulatory control for these devices. Otologists in Korea should also be interested in this issue and give their opinions on PSAPs in a proactive and responsible manner.

\section{CONFLICT OF INTEREST}

No potential conflict of interest relevant to this article was reported.

\section{ORCID}

Goun Choe https://orcid.org/0000-0002-3839-2324

Moo Kyun Park https://orcid.org/0000-0002-8635-797X

\section{AUTHOR CONTRIBUTIONS}

Conceptualization: MKP. Data curationy: GC. Formal analysisy: GC. Methodology: GC. Project administration: MKP. Visualization: MKP.Writing-original draft: GC.Writing-review \& editing: MKP.

\section{REFERENCES}

1. Mamo SK, Reed NS, Nieman CL, Oh ES, Lin FR. Personal sound amplifiers for adults with hearing loss. Am J Med. 2016 Mar;129(3): 245-50.

2. Reed NS, Betz J, Kendig N, Korczak M, Lin FR. Personal sound amplification products vs a conventional hearing aid for speech understanding in noise. JAMA. 2017 Jul;318(1):89-90.

3. Brody L, Wu YH, Stangl E. A comparison of personal sound amplification products and hearing aids in ecologically relevant test environments. Am J Audiol. 2018 Dec;27(4):581-93.

4. Cho YS, Park SY, Seol HY, Lim JH, Cho YS, Hong SH, et al. Clinical performance evaluation of a personal sound amplification product vs a basic hearing aid and a premium hearing aid. JAMA Otolaryngol Head Neck Surg. 2019 Jun;145(6):516-22.

5. Choi JE, Kim J, Yoon SH, Hong SH, Moon IJ. A personal sound amplification product compared to a basic hearing aid for speech intelligibility in adults with mild-to-moderate sensorineural hearing loss. J Audiol Otol. 2020 Apr;24(2):91-8.

6. Kim GY, Kim JS, Jo M, Seol HY, Cho YS, Moon IJ. Feasibility of personal sound amplification products in patients with moderate hearing loss: a pilot study. Clin Exp Otorhinolaryngol. 2022 Feb;15(1): 60-8.

7. Reed NS, Oliver A, Srinivasan NK, Lin FR, Korczak PA. Pilot comparison of adjustment protocols of personal sound amplification products. Semin Hear. 2019 Feb;40(1):26-36.

8. Lakshmi MS, Rout A, Morris A, Smaldino J. Consumer opinion of personal sound amplification products: a preliminary sentiment analysis. Am J Audiol. 2019 Aug;28(2S):450-9. 